\title{
A propósito de los 25 años del Estatuto Orgánico de Bogotá (EOB). Tres miradas sobre el modelo de descentralización de Bogotá como un modelo híbrido*
}

\author{
Apropos 25 Years of the Bogotá Organic Statute (EOB). Three Viewpoints about the Decentralization Model in \\ Bogotá as a Hybrid Model
}

\section{Claudia Dangond Gibsone \\ Pontificia Universidad Javeriana, Colombia \\ ORCID: https://orcid.org/0000-0001-9364-5630}

Jean-François Jolly ${ }^{\mathrm{a}}$

Pontificia Universidad Javeriana, Colombia

jjolly@javeriana.edu.co

ORCID: https://orcid.org/0000-0002-0611-0548
DOI: https://doi.org/10.11144/Javeriana.papo25.paeo

Recibido: 06 Diciembre 2018

Aceptado: 25 Abril 2019

Publicado: 29 Junio 2020

\section{Resumen:}

El Estatuto Orgánico de Bogotá - EOB - fija las relaciones entre la Administración central del Distrito y sus 20 localidades, y plantea así los lineamientos de la descentralización subdistrital de Bogotá. Este artículo reúne tres miradas que surgen a partir de una investigación reciente adelantada sobre el EOB por parte del grupo de investigación interfacultades "Políticas Urbanas" de la Pontificia Universidad Javeriana. La idea principal de estas reflexiones es cómo a partir del EOB, la descentralización de Bogotá se ha visto caracterizada por elementos de diversos modelos que la llevan a ser calificada como fruto de un proceso híbrido.

Después de hacer un recorrido por los antecedentes históricos del EOB para comprender su origen, y los debates en la Asamblea Constituyente para poder dar explicación a las diversas corrientes que lo inspiraron y le dieron vida, explicitando el contexto político de la época, se presenta el diseño de la descentralización en Bogotá y cuáles son sus particularidades. Así, se hace una aproximación de la caracterización del modelo de descentralización de Bogotá como un proceso híbrido entre descentralización, desconcentración y delegación que, unas veces, privilegia el modelo económico de descentralización y, otras, el modelo político.

Las reflexiones finales dejan pensar que más que "desdibujarse y/o modificarse" bajo el efecto de los instrumentos de planificación y de gestión que le fueron posteriores, son las particularidades del "modelo de descentralización adoptado" en el EOB —es decir esencialmente su carácter híbrido. las que permiten entender lo que está ocurriendo cuando se pasa de la formulación de las directrices que se dan a nivel central del Distrito a la formulación y la implementación de los proyectos. Eso hace necesario fomentar un diálogo más fluido entre Administración central y localidades que fortalezca los espacios en los que la voz ciudadana esté presente como interlocutor válido.

Palabras clave: Estatuto Orgánico de Bogotá, descentralización, modelos de descentralización, localidades.

\section{Abstract:}

The Bogotá Organic Statute - EOB, after the Spanish acronym — set outs the relationships between the city central administration and its 20 local districts and provides the guidelines for a district-based decentralization in Bogotá. This paper gathers three viewpoints emerging from a recent research conducted by the inter-faculty research group at the Pontificia Universidad Javeriana, the "Urban Policy Group" that addressed the theme of the EOB. The main idea from these reflections is how the decentralization in Bogotá has been characterized based on the EOB, in relation to elements from different models allowing defining it as the result from a hybrid process. After a review of the EOB historic background in order to understand its origin, and, having explored the debates in the Constitutional Assembly in order to explain the different trends that inspired and brought it into existence as well as the political context back then, this paper provides a design of the decentralization in Bogotá and its particularities. This way, the characterization of the decentralization model in Bogotá is described as a hybrid process between the decentralization, deconcentration and delegation that sometimes favors the decentralization economic model and, sometimes, the political model. The final reflections lead to think that instead of "becoming blurred and/or modified" due to the effect of the planning and management devices that appeared later, it is the particularities of the "decentralization model adopted in the EOB" -i.e., its largely hybrid nature - that allow understanding what happens when going from the guidelines produced by the city central administration to the formulation and implementation of the projects. This fact requires encouraging a more fluent dialogue between the central administration and the local districts to strengthen the spaces where the citizen's voice is present as a valid speaker.

Notas de autor

a Autor de correspondencia. Correo electrónico: jjolly@javeriana.edu.co 
Keywords: Bogotá Organic Statute, decentralization, decentralization model, local districts.

\section{Introducción}

El presente artículo ${ }^{1}$ es de carácter mixto o, si se quiere, "híbrido". Si bien se apoya en algunos resultados y en el mismo proceso de una investigación adelantada por el grupo de investigación interfacultades "Políticas Urbanas" de la Pontificia Universidad Javeriana o "Grupo PU” sobre el Estatuto Orgánico de Bogotá $\mathrm{EOB}^{2}$-, no constituye una presentación con el esquema clásico de un “artículo de investigación”. Tampoco corresponde estrictamente a un “artículo de reflexión”. Más bien, aprovechando los 25 años de la publicación del EOB, presenta tres miradas sobre el modelo de descentralización de Bogotá como un modelo híbrido.

Para el efecto, se procederá así:

En la primera mirada, se hace un recorrido por los antecedentes históricos del Estatuto Orgánico de Bogotá (Gobierno de Colombia, 1993) y los debates que se dieron a su propósito en la Comisión II de la Asamblea Constituyente, para así comprender su origen y poder dar explicación a las diversas corrientes que lo inspiraron y le dieron vida.

En la segunda mirada, se analiza el EOB y las posteriores disposiciones normativas como un híbrido entre descentralización $y$ desconcentración con delegación.

La tercera mirada se concentrará en hacer, a partir de un análisis de las posiciones de dos entidades del Distrito, la Secretaría de Hacienda y la Secretaría de Gobierno, una aproximación hacia una caracterización del modelo de descentralización de Bogotá como un proceso que, unas veces, privilegia el modelo económico de descentralización y, otras, el modelo político.

Finalmente, se aportarán algunas reflexiones finales sobre el carácter a la vez híbrido e inestable del EOB, y la necesidad de fomentar un diálogo más fluido entre Administración central y localidades, que fortalezca los espacios en los que la voz ciudadana esté presente como interlocutor válido.

\section{Los antecedentes y los debates en la constituyente sobre el estatuto orgánico de Bogotá}

Es importante señalar que, en el caso de Bogotá, la Constitución de 1991 (Asamblea Nacional Constituyente, 1991a) es un hito en la medida en que "consolida" un proceso que viene de años atrás y que se remonta al año 1954, cuando, durante el gobierno del General Rojas Pinilla, se expide el Decreto 3640 (Gobierno de Colombia, 1954). Con este decreto, como bien lo indica Vásquez, citado por Botero y Suárez (2010),

se dividió administrativamente la ciudad en zonas, con un esquema de desconcentración, en el cual cada zona tiene un alcalde menor, con funciones de inspector de policía, y quien cumple la tarea de 'ser simples subalternos y representantes del Alcalde Mayor en sus respectivos territorios'.

Con los años, el proceso fue evolucionando, y se fueron introduciendo esquemas de participación ciudadana y medidas que fueron extendiendo la autonomía local, al punto que para 1982 ya se contaba con 19 zonas, cada una con un alcalde local menor que tenía la facultad de diseñar un plan para sus zonas, con la asesoría del Departamento Nacional de Planeación (Botero y Suárez, 2010, p. 17).

Con el objeto de profundizar la descentralización en el país, y bajo la premisa de que el municipio es la célula básica del ordenamiento territorial, el $1^{\circ}$ de enero de 1986 se reforma la Constitución Política, y mediante el Acto Legislativo 01 (Gobierno de Colombia, 1986a) se aprueba la elección popular de alcaldes en todo el país.

Esta importante reforma a la Constitución de 1886 deja entrever la necesidad de contar con un Código de Régimen Municipal, y se expide el Decreto 1333 de abril 25 de 1986 (Gobierno de Colombia, 1986b), cuyo objeto, según Lordello de Melo, citado por Botero y Suárez (2010, p. 17), era la promoción de la "descentralización interna de la administración municipal, al mismo tiempo que se adelantan prácticas de 
participación ciudadana”. El Código previó la creación de Juntas Administradoras Locales —JAL_ y de alcaldías locales con funciones de mayor responsabilidad.

En desarrollo del Código se promulgó el Acuerdo 8 de 1987 (Alcaldía de Bogotá, 1987) que creó 20 JAL elegidas por voto popular en cada una de las zonas de Bogotá, pero que fue anulado por el Tribunal Administrativo de Cundinamarca lo que, como bien lo indican Botero y Suárez (2010, p. 18), dejó a la ciudad en un "limbo jurídico" y en una situación precaria respecto a la coordinación entre el nivel distrital y el nivel local en materia de planeación, de prestación y de control de los servicios públicos.

Del contexto normativo y político anterior va a surgir la visión descentralizadora planteada por la Carta Política de 1991 (Asamblea Nacional Constituyente, 1991a). Es a partir de ella que se empieza a vislumbrar una salida a la situación indefinida en la que se encontraba la capital colombiana, y se empieza a "consolidar" el modelo de descentralización de Bogotá sobre la base de unas premisas presentadas por algunos de los constituyentes de la época.

Es así como para Carlos Lemos Simmonds (1991) el problema de la movilidad, que ya se vislumbraba en una ciudad con cerca de siete millones de habitantes, debía ser abordado mediante el fortalecimiento de las instituciones locales y la disposición de importantes recursos financieros. Mientras tanto, Jaime Castro Castro (1991) consideraba que era necesario contar con una administración eficiente y eficaz, para lo cual se requería "descentralizar la prestación de los servicios públicos" hacia las zonas en las que se dividiría la ciudad.

En general, los miembros de la Comisión II de la Asamblea Constituyente, encargados del tema territorial, como lo recuerdan Botero y Suárez (2010, p. 26), sostenían que "era de vital importancia que Bogotá D. C., tuviese 'la competencia legislativa en asuntos fiscales, única vía para resolver la carga financiera creada por la urbanización acelerada en la sabana de Bogotá".

Ahora bien, un breve análisis de los debates que tuvieron lugar entre los constituyentes de la Comisión II permite evidenciar el "acuerdo a medias" y, por ende, el carácter híbrido de la descentralización de la ciudad capital que resultara más tarde de este acuerdo a medias. Esto se puede comprobar con las explicaciones que dos de ellos, que fueron alcaldes de la ciudad, ofrecen en una entrevista que les hizo la politóloga María Angélica Nieto en un trabajo que realizó en el marco del Grupo PU.

Así, mientras para Jaime Castro Castro

...eso fue un debate que hubo en la Asamblea, porque había propuestas en el sentido de que se eligieran popularmente y había propuestas para que el Alcalde Mayor los nombrara y renovara libremente. Entonces se optó un mecanismo intermedio que es una elección de segundo grado, una fórmula mixta. Es una elección popular indirecta porque la terna la elaboran quienes han sido elegidos popularmente. ...Se consideró que una elección popular, en ese momento de 20 alcaldes locales, descuadernaba la ciudad. No permitía un mecanismo fácil de coordinación y de integración, y que cada alcalde local hiciera lo que quisiera; no tenía sujeción frente al alcalde vecino. Además, no había una identidad política, ni una historia como lo tienen los municipios donde se eligen alcaldes. Una cosa es elegir el alcalde de Tunja, que tiene tanta vida e identidad política como la de Bogotá, y otra es elegir el alcalde, por ejemplo, de Barrios Unidos donde no hay el concepto de identidad política. (Nieto, 2008, pp. 8-9).

\section{Para Augusto Ramírez Ocampo}

...Él [Jaime Castro] quería hacer lo mismo que yo había hecho cuando yo había sido alcalde: fortalecer las alcaldías zonales, para darles verdadera importancia; pero él quería que los alcaldes zonales fueran elegidos popularmente. Yo no era partidario de eso y me opuse rotundamente, por algunas observaciones muy pragmáticas que había visto en otras ciudades. Porque me había parecido muy difícil que, si nosotros desvertebrábamos la administración de la ciudad, descuadernaba a la ciudad, eligiendo popularmente a los alcaldes locales, le quitaba unicidad a la administración pública. Eso creaba una situación imposible, por ejemplo, en el plan vial, en los servicios públicos, que eran muy difíciles de administrar. Yo sí me opuse decididamente a que los alcaldes locales fueran elegidos popularmente. (Nieto, 2008, pp. 8-9)

De estas dos posiciones opuestas discutieron intensamente los miembros de la Comisión II hasta que, finalmente, llegaron a lo que podría llamarse un "acuerdo a medias" o, en términos del mismo Augusto Ramírez Ocampo, a una “fórmula de transacción” según la cual 
...se elegían los ediles popularmente, y que los ediles pasaran una terna a la consideración del Alcalde Mayor y este mismo nombraba los alcaldes locales y, por lo tanto, podía cambiarlos. Yo creo que esa fórmula quedó bien desde el punto de vista político, entre otras cosas, el hecho de exigir una terna significa que casi siempre hay propuestas de distintos partidos políticos porque los ediles pertenecen a distintos partidos, entonces pueden presentarse opciones a los alcaldes y los alcaldes tienen la palabra definitiva. (Nieto, 2008, pp. 9-10)

Vale la pena recordar que el punto de partida del régimen descentralizado de la capital colombiana es la expedición y entrada en vigor de la Constitución Política de 1991 (Asamblea Nacional Constituyente, 1991a), por cuanto esta consagra un régimen unitario pero descentralizado ${ }^{3}$ en el que Bogotá, por ser la capital, aparece con un régimen especial. Si bien Colombia ha sido, en distintos momentos de su historia, un Estado Unitario con régimen descentralizado, es en 1991 que dicha premisa se consolida.

En cuanto a Bogotá, la norma originalmente aprobada ${ }^{4}$ por la Asamblea Nacional Constituyente decía:

ART. 322. Santa Fe de Bogotá, Capital de la República y del Departamento de Cundinamarca, se organiza como Distrito Capital.

Su régimen político, fiscal y administrativo será el que determinen la Constitución, las leyes especiales que para el mismo se dicten y las disposiciones vigentes para los municipios.

Con base en las normas generales que establezca la ley, el concejo a iniciativa del alcalde, dividirá el territorio distrital en localidades, de acuerdo con las características sociales de sus habitantes, y hará el correspondiente reparto de competencias y funciones administrativas.

A las autoridades distritales corresponderá garantizar el desarrollo armónico e integrado de la ciudad y la eficiente prestación de los servicios a cargo del Distrito; a las locales, la gestión de los asuntos propios de su territorio. (Asamblea Nacional Constituyente, 1991b)

Igualmente indicó el Constituyente de 1991 que

...si durante los dos años siguientes a la fecha de promulgación (de la Constitución), el Congreso no dicta la ley a que se refieren los Artículos 322, 323 y 324, sobre régimen especial para el Distrito Capital de Santa Fe de Bogotá, el Gobierno por una sola vez expedirá las normas correspondientes. (Asamblea Nacional Constituyente, 1991b, Art. Transitorio 41).

\section{La descentralización de Bogotá: un híbrido entre descentralización y desconcentración con delegación}

Finalizada la Asamblea Constituyente, y como bien lo indica un análisis de la Ley 1 de enero 28 de 1992 (Gobierno de Colombia, 1992), del Estatuto Orgánico de Bogotá (EOB) y de algunas disposiciones posteriores a su publicación, siguieron en otros espacios unos "tire y afloje" entre partidarios de una descentralización "a la Jaime Castro Castro" y partidarios más bien de una desconcentración con delegación "a la Augusto Ramírez Ocampo”.

\section{La Ley 1 de enero 28 de 1992 y el Estatuto Orgánico de Bogotá (EOB)}

En esta perspectiva, y con la expedición de la Ley 1 de enero 28 de 1992 "por la cual se provee a la organización y funcionamiento de las Juntas Administradoras Locales en el Distrito Capital” (Gobierno de Colombia, 1992), el Congreso de la República reguló los primeros desarrollos de la organización político-administrativa de la ciudad, y presentó los criterios con los cuales la Capital debía tener una división territorial atendiendo las características sociales de los habitantes:

Artículo $1^{\circ}$. El Concejo Distrital, a iniciativa del Alcalde Mayor, dividirá el territorio del Distrito Capital en localidades urbanas y rurales, de acuerdo con las características sociales de sus habitantes y realizará el correspondiente reparto de competencias y funciones, con el fin de propiciar la organización y participación de la comunidad de la gestión, prestación 
y administración de los servicios públicos; la realización de las obras de infraestructura local y en general, los programas de beneficio comunitario. (Diario Oficial, 1992)

En palabras de Velásquez Gavilanes (2003. p. 32), los principios que informan esta ley son:

1. La gestión de las autoridades centrales y locales puede ser concurrente, subsidiaria o complementaria, 2. La distribución de funciones debe buscar la eficiencia, la participación y la armonía entre los planes centrales y locales, 3. Para que puedan desempeñarse, las funciones deben asignarse acompañadas de recursos.

Fue esta misma ley la que señaló en su artículo $6^{\circ}$ que "las Juntas Administradoras Locales son corporaciones públicas que se elegirán popularmente para períodos de tres años”, y que le dio la facultad al Concejo de Bogotá para determinar, de acuerdo con la población de cada localidad, el número de ediles de cada JAL, indicando que, en todo caso, no debería ser inferior a siete. Así mismo, estableció un período fijo de tres años para loa alcaldes locales, período durante el cual se les dio autonomía para la destinación de los recursos públicos, previa aprobación de las JAL (Gobierno de Colombia, 1992, Artículo 30). Igualmente, como bien lo señalan Botero y Suárez (2010, p. 18), la ley estableció un período fijo para los alcaldes de cada localidad y señaló los requisitos para ser tanto edil como alcalde local.

Para efectos de proceder con la ejecución de los recursos destinados a las localidades, el Artículo 37 de la Ley 1 de 1992 (Gobierno de Colombia, 1992) creó para cada una de ellas un "Fondo de desarrollo para la financiación de la prestación de los servicios y la construcción de las obras" con recursos provenientes del presupuesto de la administración del Distrito Capital que, según el Artículo 35 (Gobierno de Colombia, 1992), son asignados a cada localidad de acuerdo con las necesidades básicas insatisfechas de la población, según los índices que para el efecto estableciera el Departamento Administrativo de Planeación Distrital. En términos de la Ley 1 de 1992 (Gobierno de Colombia, 1992), la representación legal de cada fondo estaba en cabeza el respectivo Alcalde Local, y la Junta Directiva correspondía a la correspondiente JAL, con lo cual, la autonomía que se pregonaba desde la Carta Política iba teniendo un desarrollo normativo y práctico.

La implementación de la Ley 1 de 1992 (Gobierno de Colombia, 1992) correspondió al Alcalde Jaime Castro Castro, quien fue Alcalde Mayor entre del $1^{\circ}$ de junio de 1992 y el 31 diciembre de 1994. Fue precisamente en esa administración que se detectaron algunos problemas que, de acuerdo con los expertos de la época, provenían de la mayor autonomía otorgada a las divisiones territoriales de la ciudad.

Fue así como, con el objeto de realizar ajustes al régimen especial de Bogotá e, con el fin de controlar a los alcaldes menores (locales) así como a las JAL, es decir, de recortar la autonomía de las localidades, se presentó al Congreso de la República un proyecto de estatuto para la ciudad Capital.

Como el Congreso no lo tramitó oportunamente, el 21 de julio de 1993, el Presidente de la República, César Gaviria Trujillo, expidió el Decreto 1421 "por el cual se dicta el régimen especial para el Distrito Capital de Santa Fe de Bogotá” (Gobierno de Colombia, 1993), es decir, el Estatuto Orgánico de Bogotá5. Según la exposición de motivos, dicho estatuto buscó "darle vida y operatividad a ese régimen especial en el marco de la descentralización de su territorio y hacer de la capital una ciudad moderna, capaz de garantizar a sus ciudadanos la prestación eficiente y efectiva de los servicios públicos". Pero cabe anotar que el EOB recortó en algún grado la autonomía que originalmente tuvieron las localidades, es decir, recentralizó algunas funciones de las que ellas habían sido revestidas por la Ley 1 de 1992 (Gobierno de Colombia, 1992). Con este recorte de "independencia", lo que se quería en realidad era aminorar las dificultades que habían surgido inicialmente.

En efecto, entre las modificaciones más relevantes está la posibilidad del Alcalde Mayor de no solamente nombrar libremente a los alcaldes locales, sino también de removerlos, de acuerdo con su desempeño. Así mismo, con la idea de evitar y controlar hechos de corrupción y desvío de recursos, el gasto público se recentralizó, y las localidades perdieron autonomía; en ese sentido, los alcaldes menores (locales) dejaron de ser los representantes legales de los fondos, y esa función pasó a ser responsabilidad del Alcalde Mayor. Desde ese momento, solo este último tuvo la facultad de ordenar el gasto y las inversiones para las localidades. 
Pero el Estatuto no se quedó en recortes y limitaciones a la autonomía. Como bien lo anotan Botero y Suárez (2010, p. 20-27), es cierto que también buscó que existiera un mayor diálogo y una mayor coordinación entre el nivel central y el nivel local de la administración de la ciudad, para lo cual las funciones de las localidades fueron más específicas.

Para Botero y Suárez (2010, p. 21), las modificaciones, que desde el punto de vista normativo y político se han realizado, han mostrado que la descentralización del régimen de Bogotá, Distrito Capital, se ha enfocado más desde la perspectiva de la eficiencia que desde el ejercicio real del poder local. En la perspectiva que se desarrollará más tarde en este artículo, es posible decir que el EOB se inclinó finalmente más hacia el modelo económico que hacia el modelo político de descentralización.

A lo anterior debe agregarse que la asignación de recursos del presupuesto distrital a las localidades fue modificada con la entrada en vigor del EOB. En efecto, la Ley 1 de 1992 (Gobierno de Colombia, 1992) preveía que

...no menos del diez por ciento (10\%) de los ingresos del presupuesto de la administración central del Distrito Capital se asignará a las localidades, porcentaje que se incrementaría anual y acumulativamente en un dos por ciento (2\%) hasta alcanzar como mínimo el veinte por ciento (20\%) en la vigencia fiscal de mil novecientos noventa y ocho. (Art. 30)

No obstante, posteriormente se estableció que esos recursos que se transferían podrían ser del $20 \%$ o menos del presupuesto distrital, poniendo como límite el 10\% de los ingresos corrientes de la administración central. En todo caso, como se vio atrás, la disposición de tales recursos estaría a cargo del Alcalde Mayor como ordenador del gasto.

Para la Secretaría de Gobierno Distrital, citada por Botero y Suárez (2010, p. 30), el Estatuto de Bogotá de 1993 recoge un modelo de descentralización territorial que "establece lo respectivo a los objetivos, composición, funcionamiento, calidades de los funcionarios y competencias de las localidades, las Juntas Administradoras Locales y los Alcaldes Locales".

Para algunos, este recorrido hace que el modelo de descentralización de Bogotá sea sui generis en la medida en que "recoge matices de distintas corrientes descentralizadoras acomodadas y ensambladas con elementos de procesos de desconcentración y delegación” (Botero y Suárez, 2010, p. 18, cursivas añadidas), y en que trata de dar una respuesta a las necesidades de gobierno, administración y servicio que demanda la ciudad y sus localidades.

Según Botero y Suárez (2010, p. 18), las particularidades que se derivan de lo dicho anteriormente se pueden enumerar así:

Consagración de un modelo de descentralización para la ciudad de Bogotá en el que se pregona la autonomía de las localidades.

Descentralización territorial.

Necesidad de generar desarrollo integral sin desconocer las diferencias en materia de necesidades entre las localidades.

Designación del alcalde menor (local) de cada localidad por parte del Alcalde Mayor de la ciudad para período fijo; no obstante, el Alcalde Mayor tiene la facultad de libre remoción.

Existencia de cuerpos colegiados representativos de los intereses de los habitantes de cada localidad (Juntas Administradoras Locales — JAL_ que sí son de elección popular.

\section{Algunas disposiciones posteriores a la publicación del EOB (1993-2007)}

Si bien el modelo de descentralización de Bogotá, estructurado a partir de la expedición de la Constitución de 1991 (Asamblea Nacional Constituyente, 1991a), estaba concebido para otorgar a las localidades la autonomía suficiente para que sus autoridades, unas nombradas por el Alcalde Mayor (alcaldes menores, locales), y otras elegidas popularmente (ediles), pudieran decidir y disponer la estrategia de desarrollo de acuerdo con las necesidades de cada uno de esos territorios, es posible afirmar que, en la práctica, las distintas administraciones de la ciudad fueron tomando ciertas medidas que vinieron matizar la idea original de ese modelo. 
Tras la publicación del EOB, siguieron los debates, que terminaron por su no aprobación por el Congreso y su posterior publicación por Decreto. Siguió siendo la columna vertebral de la acción de las administraciones que gobernaron la ciudad entre 1993 y 2007, que han producido los desarrollos normativos significativos siguientes:

Jaime Castro Castro (1992-1994). Considerado como el "padre del EOB", y buscando concretar la autonomía de las localidades, al utilizar el ejercicio de la figura de la delegación, la administración de Jaime Castro Castro radicó, ante las alcaldías locales, la función de contratar y de ordenar el gasto con cargo a los Fondos de Desarrollo Local mediante el Decreto Distrital 533 de 1993 (Alcaldía de Bogotá, 1993a) y los Decretos Distritales 698 de 1993 (Alcaldía de Bogotá, 1993b) y 50 de 1994, respectivamente (Alcaldía de Bogotá, 1994).

Antanas Mockus-Paul Bromberg (1995-1997). Con el Plan de Desarrollo Formar la Ciudad ${ }^{6}$, esta administración buscó profundizar la descentralización fortaleciendo los canales de participación a través de las veedurías ciudadanas, propendiendo para que la población pudiera tener injerencia en las políticas públicas que determinara el gobierno de la ciudad. Para el efecto, organizó los llamados "encuentros ciudadanos".

Enrique Peñalosa (1998-2000). La administración de Enrique Peñalosa abordó el fortalecimiento de la descentralización a partir del discurso que sostenía la necesidad de trabajar por la democracia participativa y ciudadana. De esta manera, continuó con la apertura de espacios de diálogo con la ciudadanía, tarea que había sido iniciada por la primera administración de Antanas Mockus. En ese sentido, el Decreto Distrital 739 de 1998 (Alcaldía de Bogotá, 1998a) fijó las directrices para adelantar los Planes de Desarrollo Locales con metodología participativa en espacios especialmente diseñados para ello, siguiendo con los encuentros ciudadanos, lo cual pudo hacerse sobre la base del Decreto Distrital 518 de 1999 (Alcaldía de Bogotá, 1999), que otorgaba competencia a los alcaldes locales para organizar los correspondientes encuentros ciudadanos, atendiendo las particularidades de cada localidad.

Sin embargo, y en oposición a la medida anterior, el Decreto 176 de 1998 (Alcaldía de Bogotá, 1998b) creó las Unidades Ejecutivas Locales (UEL), a las que se les otorgó la tarea de centralizar el gasto que se les había dado con anterioridad a los alcaldes locales. Así, esta administración recogió las competencias que desde la gestión del alcalde Castro se habían entregado a las localidades, encabezadas por los alcaldes, y que posteriormente se centralizaron en la función de contratar con cargo a los Fondos de Desarrollo Local.

Para atenuar esa evidente pérdida de autonomía de las localidades y de sus autoridades, les fueron delegadas las siguientes competencias, resumidas por Botero y Suárez (2010, p. 25) así: "coordinación de la acción administrativa del Distrito en las localidades. Coordinación de la gestión administrativa asignada a las comisarías de familia, inspecciones de policía, casas de justicia y al Programa de Mediación y Conciliación".

Antanas Mockus (2001-2003). En el Plan de desarrollo económico, social y de obras públicas 2001-2004, Bogotá para vivir todos del mismo lado, de la segunda administración de Antanas Mockus, nuevamente se encuentra la idea de hacer realidad la participación ciudadana. Con este propósito, se crearon con el Decreto 098 de 2002 (Alcaldía de Bogotá, 2002) los Consejos Locales de Gobierno, conformados por actores del nivel central y del nivel local para permitir un diálogo permanente y, por tanto, una mayor coordinación.

A los alcaldes locales, considerados como dependencias de la Secretaría de Gobierno, les fue delegada, por el Decreto Distrital 367 de 2001 (Alcaldía de Bogotá, 2001), la representación judicial y extrajudicial de los Fondos de Desarrollo Local (Botero y Suárez, 2010, p. 25).

Luis Eduardo Garzón (2004-2007). Con el Plan de Desarrollo Bogotá sin Indiferencia, un compromiso social contra la pobreza y la exclusión y disposiciones ulteriores, la administración de Luis Eduardo Garzón trabajó en el fortalecimiento administrativo de las localidades, todo con el objeto de permitir que el acercamiento con la ciudadanía se produjera sobre bases más sólidas y permanentes. Así, se expidió el Decreto Distrital 124 de 2005 (Alcaldía de Bogotá, 2005) "por medio del cual se reorganizan los Consejos Locales de Gobierno, y se dictan otras disposiciones", donde se hace evidente la priorización de algunos territorios dentro de la ciudad.

En esa misma línea, se dictaron el Decreto Nacional 1350 (Gobierno de Colombia, 2005) y el 342 de 2006 (Alcaldía de Bogotá, 2006a), de carácter distrital, referidos a la manera en que debían ser postulados los candidatos de las ternas de las que saldría cada uno de los alcaldes locales.

El Concejo de Bogotá aprobó el Acuerdo 257 de 2006 (Alcaldía de Bogotá, 2006b) “por el cual se dictan normas básicas sobre la estructura, organización y funcionamiento de los organismos y de las entidades de Bogotá, Distrito Capital, y se expiden otras disposiciones" que, de acuerdo con Botero y Suárez (2010, p. 24), "definió las acciones administrativas respecto a descentralización funcional, desconcentración, delegación y asignación".

Luego, la administración expidió el Decreto Distrital 539 de 2006 (Alcaldía de Bogotá, 2006c) que, en función de lo expresado por el Acuerdo 257 (Alcaldía de Bogotá, 2006b), puntualizó sobre las funciones de 
algunas secretarías de la Alcaldía Mayor, de tal manera que la coordinación pretendida entre el nivel local y distrital fuera una realidad.

Con el desmonte progresivo de las UEL, nuevamente se abrió la posibilidad para que los alcaldes locales, a partir de una delegación hecha por la Alcaldía Mayor, pudieran contratar y ordenar el gasto con miras a pagar los programas y subprogramas contemplados en los planes de desarrollo local, todo con cargo a los Fondos de Desarrollo local. Esto se refleja en el Decreto Distrital 421 de 2004 (Alcaldía de Bogotá, 2004).

Este breve recorrido hecho por las diferentes administraciones que ha tenido Bogotá, a partir de la expedición del EOB y hasta 2007, muestra bien las variadas reformas, interpretaciones y alcances que le han querido dar y que han sido expresadas y plasmadas en instrumentos de política pública, pero sobre todo en los Decretos Distritales y los Acuerdos expedidos por los alcaldes y concejos de turno. Dichas disposiciones terminaron en fortalecer el híbrido entre descentralización y desconcentración con delegación.

En otras palabras, es posible concluir que de la idea de una ciudad descentralizada en la que la autonomía de las localidades se refleja no solo en la organización administrativa sino también en la posibilidad de que estas últimas puedan disponer de los recursos necesarios - de acuerdo con las necesidades detectadas, desde la vigencia del Estatuto Orgánico de 1993 (Gobierno de Colombia, 1993) - se ha pasado a una fluctuación entre una descentralización moderada, una desconcentración de funciones y una recentralización funcional y de recursos económicos.

Pero existe otra razón por la que puede afirmarse que la descentralización de Bogotá tiene un carácter híbrido: el hecho de que hay elementos que más que tener carácter descentralizador, corresponden a lo que es la desconcentración como tal. En efecto, el hecho de que el Alcalde Mayor les entregue a los alcaldes menores (locales) algunas de sus funciones, bien porque lo decida o porque el Concejo así lo disponga, sin entregarles autonomía financiera, es propio de la desconcentración. Ni siquiera puede afirmarse hoy que los alcaldes locales tengan un período fijo en cuanto el Alcalde Mayor tiene la posibilidad de removerlos (bajo el criterio de calificación de su eficiencia) en cualquier momento; es decir, más que autoridades autónomas, estos funcionarios son subalternos que deben cumplir con lo dispuesto por el Alcalde Mayor.

Son, además, dependientes financieramente y, en esa calidad, no son pocas las ocasiones en las que más que invertir los recursos en proyectos propios de las localidades, y también sucede que deben destinarse a la cofinanciación de los proyectos del distrito (Botero y Suárez, 2010, p. 31). El hecho de que no exista la posibilidad de que las autoridades de las localidades dispongan y ejecuten autónomamente los recursos de los fondos de desarrollo local limita radicalmente el régimen descentralizado.

En ese sentido, la creación de las UEL chocaba con la existencia de la JAL, en el sentido en que a través de ellas el Alcalde Mayor define la prioridad del gasto en cada localidad; lo que, en muchos casos, como lo señalan Botero y Suárez (2010, p. 22), genera que "las prioridades y problemáticas de la localidad y de la comunidad se ven disociadas porque no tiene autonomía”.

A pesar de que en Colombia se establece un régimen descentralizado en el Estado Unitario, y que Bogotá, como ciudad capital, es de las entidades territoriales que más goza de autonomía, sus propias autoridades han hecho de la ciudad una menos descentralizada de lo que el mismo Constituyente previó en 1991, y ciertamente menos autónoma de lo que en 1993 se estipuló en el Estatuto Orgánico de la ciudad (Gobierno de Colombia, 1993).

En efecto, como lo afirman Botero y Suárez (2010, p. 29), los desarrollos introducidos en Bogotá han derivado en

...un proceso descentralizador para la ciudad que no generó unas localidades sólidas, puesto que estas, a pesar de ser entes descentralizados territorialmente, dependían del sector central de la ciudad en materia administrativa - las plantas de personal de las localidades dependían de la Secretaría de Gobierno Distrital y los alcaldes locales son designados por el Alcalde Mayor de la Ciudad- y en materia financiera, puesto que los recursos de las localidades tenían como ordenador del gasto al Alcalde Mayor de la ciudad. 
Entonces, es posible afirmar que el proceso de descentralización de Bogotá, más que profundizarse, se ha ido debilitando de alguna manera, en cuanto se ha matizado con medidas y rasgos propios de la desconcentración y aún de la delegación, al punto que hoy no son claras las competencias y responsabilidades de las autoridades locales.

\title{
El EOB como modelo híbrido entre el modelo económico y el modelo político de descentralización
}

\author{
Como bien lo señaló Jaime Castro Castro en entrevista concedida a la politóloga Nieto (2008):
}

Arranca la descentralización con mucha voluntad política de parte mía, cuando empezamos forzamos la división de las localidades eran antiguas zonas, Bogotá se dividía en zonas numeradas, si esperábamos a hacer un trazado racional de las localidades, la descentralización no arrancaba nunca. Aquí ya hay una división en zonas, convirtámoslas en localidades, ganemos tiempo porque o si no, se aplaza indefinidamente. ... Nos dábamos contra las paredes porque no sabíamos cómo era el tema. Yo encontré que a las localidades se les daba el $2 \%$ y ordené que se le diera el $10 \%$ y se quedó congelado en el $10 \%$ aunque la ley permite ir hasta el $20 \%$.

Lo que indica el que se considera como el "padre del EOT" es bien significativo en cuanto al trecho que hay entre las intenciones y la manera de hacerlas realidad. Pero también plantea un problema conceptual. De hecho, normalmente, cuando se habla de descentralización, esta se predica de la relación entre nación y entidad territorial. En el caso de la ciudad de Bogotá, las localidades no son entidades territoriales, sino meras unidades descentralizadas.

Sin embargo, el Grupo PU, en su investigación Coherencia..., ha considerado que, a pesar de esta consideración fundamental, sí era posible "aplicar" a la descentralización sub distrital (es decir, entre el nivel central del Distrito y sus 20 localidades), lo que Jolly (2010, p. 43-86), en su "Marco de análisis de la descentralización en Colombia", planteó para lo que él llamó "el modelo híbrido de descentralización en Colombia”.

En esta perspectiva, para Fernando Rojas, citado por Jolly (2010, p. 61-62), quien reflexiona sobre la descentralización en América latina, fue necesario

...equilibrar realidad política y realidad administrativa con la nueva distribución de poder generada por la descentralización, y es lo que ha producido "un curioso bíbrido de 'descentralización delegada', un punto de encuentro confuso entre las formas francesa y australiana de Estado", de tal manera "que el seguimiento y la coordinación intergubernamental tomó el paso sobre la autonomía y el accountability (responsabilidad) individual” (cursivas agregadas).

Retomando, pues, esta idea de Rojas, Jolly (2010, p. 63) considera que el carácter híbrido del proceso de descentralización en Colombia tiene un triple sentido:

Motivos híbridos por implementar el proceso, por razones "económicas" como son: disminuir la presión fiscal, buscar más eficiencia en la prestación de los servicios, captar mejor las preferencias de los ciudadanos-consumidores, poner en marcha "políticas neoliberales", lucha contra el deterioro de la distribución de los ingresos, poner en marcha "agendas sociales" con enfoques asistenciales, hacer que las inversiones se adapten a las necesidades de la población ${ }^{7}$, etc., se mezclaron con razones "políticas": mejorar la gobernabilidad, fomentar la democracia participativa, superar rasgos autoritarios, lograr la paz, terminar con los paros cívicos, etc.

Referenciales ${ }^{8}$ híbridos, porque aquellos que inspiran las medidas tomadas a partir de estas razones son radicalmente diferentes y opuestos — federalismo fiscal para el "modelo económico" frente a democracia participativa para el "modelo político"- y porque la simultaneidad y la sucesión de las reformas adelantadas hacen que cohabiten disposiciones a menudo contradictorias, aunque a veces compatibles. A una sola política pública le pueden corresponder medidas cuyo referencial puede ser diferente, e incluso medidas que combinan los dos referenciales del modelo político y del modelo económico.

Combinación de fuerzas opuestas, finalmente, con fuerzas en pro de la descentralización, partidarias de la democracia participativa y la "resistencia velada" del gobierno nacional y de los partidos políticos nacionales que hace que se trate de una 
"descentralización controlada y vigilada", en otras palabras, de una "descentralización delegada" y un proceso inestable, en la búsqueda de un equilibro entre realidad política y realidad administrativa, un lado, y nueva redistribución del poder, del otro.

Así, la característica híbrida del proceso de descentralización de Bogotá, Distrito Capital, está dada no solamente por la manera cómo éste se mezcla con elementos de la desconcentración y de la delegación, sino también porque la evolución y la implementación del régimen se ha dado entre dos modelos que persiguen propósitos diferentes: el modelo económico, que busca la eficiencia en la prestación de ciertos bienes públicos, y el modelo político, donde el "gobierno local" es, según la palabra de Andrew Nickson (1998), "el vocero de los intereses compartidos (shared interests) de la comunidad para la elaboración de políticas públicas".

La tabla 1, construida a partir de Jolly (2010, p. 44-50), quien se apoya en Nickson, resume algunas características de ambos modelos y de sus postulados en cuanto al propósito del gobierno local, competencias, transferencias financieras, utilización de las transferencias financieras y participación de los ciudadanos.

TABLA 1.

Los postulados del modelo económico y los postulados del modelo político de descentralización

\begin{tabular}{|c|c|c|}
\hline Características & Postulados del Modelo Económico & Postulados del Modelo Político \\
\hline $\begin{array}{l}\text { Propósito del gobicrno } \\
\text { local }\end{array}$ & $\begin{array}{l}\text { El propósito fundamental del gobierno local es económico: la } \\
\text { eficiencia en la prestación de los servicios publicos }\end{array}$ & $\begin{array}{l}\text { La provisión de los servicios públicos ticne alguna } \\
\text { importancia, más no es escncial cuando se trata de definir } \\
\text { el rol de un gobicrno local. } \\
\text { Mucho más importante para es actuar como el vocero de } \\
\text { los intereses de la comunidad para la elaboración de politicas } \\
\text { públicas }\end{array}$ \\
\hline Competencias & $\begin{array}{l}\text { Las competencias del gobierno local son claramente definidas y } \\
\text { rcgidas por un estatuto de tipo legistativo: el gobicrno local tiene } \\
\text { cl rol de una administración local }\end{array}$ & Inscritas en la Constitución Nacional \\
\hline Transferencias financicras & $\begin{array}{l}\text { Las transferencias del nivel central al local se reparten con base } \\
\text { en una fórmula que toma en cuenta las necesidades y se calcula } \\
\text { con el fin de cumplir cstándares de provisión de servicios fijados } \\
\text { por decreto nacional }\end{array}$ & $\begin{array}{l}\text { El compartir responsabilidades con el gobicrno central en } \\
\text { cuanto a la provisión de servicios }\end{array}$ \\
\hline $\begin{array}{l}\text { Utilización de las } \\
\text { transferencias financieras }\end{array}$ & $\begin{array}{l}\text { El gobierno central se reserva controlar estrictamente los gastos } \\
\text { del segundo para mantencr la cstabilidad macrocconómica }\end{array}$ & $\begin{array}{l}\text { La intervención de los niveles más clevados de gobierno } \\
\text { solo será posible si los gobiernos de bajo nivel territorial } \\
\text { ya no son capaces de tomar decisiones }\end{array}$ \\
\hline $\begin{array}{l}\text { Participación de los } \\
\text { ciudadanos }\end{array}$ & $\begin{array}{l}\text { La participación política está limitada, dado el gran tamańo } \\
\text { promedio de los municipios, cl cscaso número de clegidos cn } \\
\text { relación con el número de ciudadanos y la transparencia } \\
\text { reducida del proceso administrativo local. Poco o nada se } \\
\text { promueve la participación colectiva en la formulación de las } \\
\text { políticas públicas, definidas por el nivel central. }\end{array}$ & $\begin{array}{l}\text { Un alto grado de autonomía política, a menudo plasmada } \\
\text { en la constitución escrita. } \\
\text { Lo importante es afirmarse como gobierno, más que } \\
\text { como sencillo provecedor de servicios espećficos. }\end{array}$ \\
\hline
\end{tabular}

Estas características explican que el proceso de Bogotá, en términos de descentralización, sea a la vez débil e instable por lo que está permanentemente buscando lograr un equilibrio entre la realidad política y la realidad administrativa, por una parte; y, por la otra, una nueva redistribución del poder.

De hecho, como lo afirma en su investigación desarrollada en el marco del proyecto Coherencia..., Montaño (2016, p. 48), citando apartes de la exposición de motivos del Proyecto de Ley 054 de 2015 (Cámara):

...desde que entró en vigencia el Estatuto Orgánico de Bogotá, se ha intentado modificar vía proyectos de ley en el Congreso de la República 34 veces, de las cuales solo 2 veces han pasado el primer debate. De esas 34 veces, 18 han tenido como objetivo central modificar el funcionamiento de las localidades, para hacerlas más descentralizadas.

Lo anterior toma más fuerza si se indica que, como bien lo señaló Montaño (2016), ${ }^{9}$ en el seno de la propia Administración central del Distrito, ambos modelos están defendidos y se traducen en dos posiciones opuestas, la de la Secretaría Distrital de Hacienda, defensora del modelo económico, y la de la Secretaría Distrital de Gobierno, ardiente promotora del modelo político. Estas dos posiciones pueden explicarse por el contexto institucional en el que se ubican ambas Secretarías.

Para entender la posición de la Secretaría de Hacienda, hay que recordar que el Distrito Capital se divide en un Sector Central, compuesto por 17 entidades, entre las que se encuentran las Secretarías de Despacho; y un Sector Descentralizado, representado por 54 entidades entre Fondos, Unidades de Atención Descentralizadas, Institutos, Empresas Sociales del Estado, Empresas Industriales y Comerciales del Estado, Sociedades, Empresas de Servicios Públicos y un Ente Universitario Autónomo (Universidad Distrital Francisco José de Caldas). Esto arroja 75 entidades de la Administración Distrital aparte de las 20 localidades, y cada una de estas representa a los 14 sectores ${ }^{10}$ que tiene el Distrito para coordinar su acción.

$\mathrm{Al}$ tener un modelo sectorizado de gestión, cada secretaría de despacho, es decir, cada sector, defiende y gestiona sus temas. La Secretaría Distrital de Hacienda, en cumplimiento de sus funciones de coherencia 
fiscal, vigilancia y cumplimiento de la eficiencia de la gestión y racionalización del gasto, es muy categórica en recalcar que las entidades, en cumplimiento de los principios presupuestales contenidos en el Estatuto Orgánico de Presupuesto del Distrito Capital, Decreto 714 de 1996 (Alcaldía de Bogotá, 1996), solo podrán viabilizar iniciativas que puedan ser debidamente financiadas con el presupuesto aprobado en la presente vigencia fiscal, estén previstas dentro de las proyecciones del Marco Fiscal de Mediano Plazo, y no afecten las metas de superávit primario del Distrito Capital.

Es decir, para la Secretaría Distrital de Hacienda, todas las entidades del Distrito deben propender por ejecutar los proyectos y políticas públicas que estén contenidas dentro del presupuesto aprobado para la vigencia. Si no está contemplado, se deben establecer claramente los costos fiscales de la iniciativa y la fuente de ingreso adicional generada para el financiamiento de dicho costo. Frente al tema de la descentralización, la Secretaría Distrital de Hacienda ha adoptado esta misma posición desde siempre, lo que la llevó a defender el "modelo económico" de descentralización.

TABLA 2.

Visión económica vs. visión política de la descentralización:

La Secretaría de Hacienda vs. la Secretaría de Gobierno

\begin{tabular}{|c|c|c|c|c|}
\hline \multirow{2}{*}{ Caracteristicas } & \multicolumn{2}{|c|}{ Secretaría Distrital de Hacienda/Modelo económico } & \multicolumn{2}{|c|}{ Secretaria Distrital de Gobierno/Modelo politico } \\
\hline & Postulados del Modelo Económico & Argumentos de la Secretaria de Hacienda & Postulados del Modelo Político & $\begin{array}{l}\text { Argumentos de La Secretaria de } \\
\text { Gobierno }\end{array}$ \\
\hline $\begin{array}{l}\text { Propósito del } \\
\text { gobierno local }\end{array}$ & $\begin{array}{l}\text { El propósito fundamental del gobierno local } \\
\text { es económico: la eficiencia en la prestación } \\
\text { de los servicios públicos }\end{array}$ & $\begin{array}{l}\text { Con un diagnóstio que evidencia el poos } \\
\text { conocimiento que tiene la ciudadania sobre } \\
\text { la dinámica de accoón local, zqué sentido } \\
\text { tiene que una alcaldia local haga politica } \\
\text { pública si la gente no la identifica? }\end{array}$ & $\begin{array}{l}\text { La provisión de los servicios } \\
\text { públicos tiene alguna importancia, } \\
\text { mais no es esencial cuando se trata } \\
\text { de definir el rol de un gobierno } \\
\text { local }\end{array}$ & $\begin{array}{l}\text { La descentralización no ha producido } \\
\text { todos los resultados que se esperaban } \\
\text { ni los que se propusieron sus } \\
\text { promotores, pero ha logrado los } \\
\text { suficientes para confirmar que el } \\
\text { proceso era nocersirio y tiene futuro }\end{array}$ \\
\hline Competencias & $\begin{array}{l}\text { Las competencias del gobierno local son } \\
\text { claramente definidas y regidas por un } \\
\text { staututo de tipo logislativo: ed gobiemo local } \\
\text { tiene el rol de una administración local }\end{array}$ & $\begin{array}{l}\text { Es necosario esclarecer las funciones de las } \\
\text { localidades, asi como darificar la } \\
\text { transferencia de recursos }\end{array}$ & $\begin{array}{l}\text { Mucho más importante para es } \\
\text { actuar como el vocero de los } \\
\text { intereses de la comunidad para la } \\
\text { elaboración de políticas públicas }\end{array}$ & $\begin{array}{l}\text { Es necesario fortalecer el acalde local } \\
\text { como mixima autoridad ejecutiva en } \\
\text { su territorio, precisando y acotando } \\
\text { sus funciones policivas y potenciando } \\
\text { al máximo su labor como gestor del } \\
\text { desarrollo local }\end{array}$ \\
\hline $\begin{array}{l}\text { Transferencias } \\
\text { financieras }\end{array}$ & $\begin{array}{l}\text { Las transferencias del nivel central al local se } \\
\text { reparten con base en una fórmula que toma } \\
\text { en cuenta las necosidades y se calcula con el } \\
\text { fin de cumplir estándares de provisión de } \\
\text { servicios fijados por decreto nacional }\end{array}$ & $\begin{array}{l}\text { E. Decreto Ley } 1421 \text { estipula que los } \\
\text { recursos a las localidades se asignan en no } \\
\text { menos del diez por ciento (10\%) de los } \\
\text { ingresos corrientes del presupuesto de la } \\
\text { administración central del Distrito, teniendo } \\
\text { en cuenta, ademis, las necesidades básicas } \\
\text { insatisfechas de su población }\end{array}$ & $\begin{array}{l}\text { El compartir responsabilidades con } \\
\text { el gobierno central en cuanto a la } \\
\text { provisión de servicios }\end{array}$ & \multirow{2}{*}{$\begin{array}{l}\text { La creación de instrumentos para dar } \\
\text { mayor capacidad de coordinación a } \\
\text { las localidades sobre la gestión } \\
\text { distrital en su territorio y articularlo a } \\
\text { un esquema democrático de } \\
\text { desonocentración territorial }\end{array}$} \\
\hline $\begin{array}{l}\text { Utilización de las } \\
\text { transferencias } \\
\text { financieras }\end{array}$ & $\begin{array}{l}\text { El gobierno central se reserva controlar } \\
\text { estrictamente los gastos del segundo para } \\
\text { mantener la estabilidad macrocoonómica }\end{array}$ & $\begin{array}{l}\text { A la Dirección Distrital de Tesoreria de la } \\
\text { Secretaria de Hacienda se le ha delegado el } \\
\text { mancejo de los recursos de las localidades oon } \\
\text { sujeción a las decisiones que adopte la Junta } \\
\text { Directiva del respectivo fondo }\end{array}$ & $\begin{array}{l}\text { La intervención de los niveles más } \\
\text { elevados de gobierno solo será } \\
\text { posible si los gobiernos de bajo } \\
\text { nivel territorial ya no son capaces } \\
\text { de tomar decisiones }\end{array}$ & \\
\hline $\begin{array}{l}\text { Participación de } \\
\text { los ciudadanos }\end{array}$ & $\begin{array}{l}\text { La participación politica está limitada, dado } \\
\text { el gran tamańo promedio de los municipios, } \\
\text { el ecáso número de elegidos en relación con } \\
\text { el número de cuudadanos y la transparencia } \\
\text { reducida del proceso administrativo local. } \\
\text { Poco o nada se promueve la participación } \\
\text { colectiva en la formulación de las politicas } \\
\text { públicas, definidas por el nivel central. }\end{array}$ & $\begin{array}{l}\text { Los mecanismos locales de expresión de } \\
\text { preferencias ciudadanas deben ser al menos } \\
\text { tan eficientes como los centrales. Si la } \\
\text { descentralizacón tendiera a fortalecer } \\
\text { procesos de asignación poco democriticos } \\
\text { o peor aún, corruptos- podria ser menos } \\
\text { eficiente que la efectuada través de procesos } \\
\text { centralizados. Este es un riesgo latente de } \\
\text { atomizacón del presupuesto }\end{array}$ & $\begin{array}{l}\text { Un gran grado de autonomía } \\
\text { politica a menudo plasmada en la } \\
\text { constitución escrita. } \\
\text { Afirmarse como gobierno, más que } \\
\text { como sencillo proveedor de } \\
\text { servicios especifios }\end{array}$ & $\begin{array}{l}\text { El otorgamiento de personeria } \\
\text { juridica a las localidades } y \text { la } \\
\text { adscripoún de los fonddos de } \\
\text { desarrollo local via Acuerdo }\end{array}$ \\
\hline
\end{tabular}

Fuente: Montaño (2016, p. 60)

Si la Secretaría de Gobierno tiene una posición opuesta que, como lo recuerda Diana Carolina Montaño (2016), la pone como defensora y promotora del modelo político, es porque

...por competencia, desde hace varios años, especialmente desde el año 2004, [la Secretaría Distrital de Gobierno] ha venido sistemáticamente elaborando documentos de trabajo donde ...se propone profundizar la descentralización como una alternativa viable para resolver los problemas locales y aumentar los niveles de participación y consolidación de la democracia ...con el fin de darle mayor autonomía a los alcaldes locales, dotarlos de personería jurídica, patrimonio propio y autonomía administrativa y, así, mejorar los índices de participación ciudadana. Esto tiene un impacto fiscal que ha sido difícil de calcular (énfasis agregado).

Entonces, "el impacto fiscal" es uno de los puntos de confrontación y de desacuerdo entre ambas Secretarías que, como bien lo anota Diana Carolina Montaño (2016, p. 64), tienen

...dos visiones de ciudad, cada una absolutamente válida, pero que, al contraponerlas, ha sido, es y será difícil encontrar un justo medio que las integre. Esto ha generado que no se haya podido tomar decisiones al respecto, abriendo un gran limbo que termina afectando la gobernabilidad de la ciudad (énfasis agregado).

La tabla 2, tomada de Montaño (2016, p. 60), recuerda los postulados de cada modelo y los Argumentos que usa cada Secretaría para defender su respectivo modelo. 


\section{Reflexiones finales}

Las tres miradas sobre el EOB que aparecen en este artículo convergen en reconocer el carácter a la vez híbrido e instable del proceso de descentralización en Bogotá. Las diferencias entre las posiciones opuestas que defendieron los miembros de la Comisión II de la Asamblea Constituyente, es decir, los partidarios de una descentralización "a la Jaime Castro Castro" y los partidarios más bien de una desconcentración con delegación “a la Augusto Ramírez Ocampo”, no fueron resueltas y se llegó, finalmente, a la solución de un régimen especial a cargo del Congreso.

Pero tampoco pudo ser aprobado el Estatuto por el Congreso, de tal manera que fue publicado por Decreto. Un análisis del mismo Estatuto, que sobre algunos puntos marca un receso frente a disposiciones posteriores a la Constitución de 1991 (Asamblea Nacional Constituyente, 1991a), pero anteriores al mismo como la Ley 1 de 1992 (Gobierno de Colombia, 1992), y de las posteriores disposiciones normativas que tomaron las administraciones del Distrito, muestra una clara evidencia que es, según la palabra de Fernando Rojas, un "curioso híbrido" entre descentralización y desconcentración con delegación. Una consecuencia de lo anterior podría ser que ese régimen ha venido perdiendo algunas de las características de que fue dotado desde 1991, en el sentido en que la mayor autonomía con que se habían concebido a las unidades territoriales de la ciudad ha ido perdiendo margen de maniobra.

Sin embargo, el análisis de los argumentos de dos entidades del Distrito, la Secretaría de Hacienda y la Secretaría de Gobierno, permiten a la vez matizar y complementar esta conclusión. Permite, en efecto, caracterizar el modelo de descentralización de Bogotá como un proceso que unas veces privilegia el modelo económico de descentralización y, otras, el modelo político. Más que "desdibujarse y/o modificarse" bajo el efecto de los instrumentos de planificación y de gestión que le fueron posteriores, otros resultados de la investigación Coherencia..., que realizó el Grupo PU, evidencian que son las particularidades del "modelo de descentralización adoptado" en el EOB — es decir esencialmente su carácter híbrido- las que permiten entender lo que está ocurriendo cuando se pasa de la elaboración de las directrices que se dan a nivel central del Distrito para la formulación y la implementación de los proyectos desarrollados en las localidades (lo que podría llamarse "la descentralización desde arriba") a la formulación e implementación de los mismos, ya no desde el nivel central, sino desde las propias localidades (lo que podría llamarse "la descentralización desde abajo").

Más allá de las diferencias entre las administraciones distritales, por ejemplo entre la primera de Antanas Mockus, muy próxima al "modelo político", y la primera de Enrique Peñalosa, muy orientada por el "modelo económico" (creación de las UEL), o la de Gustavo Petro, que escogió una vía mediana, siguen vigentes las eternas discusiones, tensiones y posiciones entre partidarios de los dos modelos ideales, que resumen bien las posiciones relativas de la Secretaría de Gobierno, ardiente defensora del "modelo político", y de la Secretaría de Hacienda, férrea defensora de la "hacienda pública" y de la "eficiencia del gasto social".

Una reflexión final sería que la crisis que vive el modelo descentralizador de la nación colombiana ha sido trasladada a la capital. La desconfianza en las autoridades locales, la falta de transparencia en los procesos y la tendencia centralista no han sido ajenas a la ciudad capital.

En todo caso, con mayor o menor grado de descentralización, se ve cada más necesario generar mecanismos para que las autoridades distritales establezcan un diálogo más fluido con las autoridades locales y que, a la par, se fortalezcan los espacios en los que la voz ciudadana esté presente como interlocutor válido de esas autoridades. En ese sentido, de acuerdo con Silva Bautista, citada por Botero y Suárez (2010, p. 30), se requiere plantear

...una división del trabajo entre la Administración Central (Alcaldía mayor y sus entidades adscritas) y los agentes locales (Alcaldes Locales), donde la primera tiene como función fundamental definir la política, supervisar, controlar el desarrollo de la ciudad como un todo y ejecutar aquellas obras o acciones que tengan una cobertura global, mientras los segundos están encargados de apoyar la tarea de gobierno mediante la implementación de la política, el control de cumplimiento de 
las normas, la recolección de información y la ejecución de obras y acciones en las que su naturaleza local les da ventajas comparativas importantes en términos de eficacia y eficiencia.

\section{Referencias}

Alcaldía de Bogotá. (1987, 29 de mayo). Acuerdo 8 de 1987. https://www.alcaldiabogota.gov.co/sisjur/normas/Nor ma1.jsp?i=549

Alcaldía de Bogotá. (1993a, 15 de septiembre). Decreto 533 de 1993. https://www.alcaldiabogota.gov.co/sisjur/norm as/Norma1.jsp? $\mathrm{i}=1746 \& \mathrm{dt}=\mathrm{S}$

Alcaldía de Bogotá. (1993b, 8 de noviembre). Decreto 698 de 1993. https://www.alcaldiabogota.gov.co/sisjur/norm as/Norma1.jsp?i $=1663 \& \mathrm{dt}=\mathrm{S}$

Alcaldía de Bogotá. (1994, 25 de enero). Decreto 50 de 1994. https://www.alcaldiabogota.gov.co/sisjur/normas/No rma1.jsp?i=2991\&dt=S)

Alcaldía de Bogotá. (1996, 15 de noviembre). Decreto 714 de 1996. https://www.veeduriadistrital.gov.co/sites/defau lt/files/files/empalme/1_2_2_\%20Estatuto\%20Org\%C3\%A1nico\%20de\%20Presupuesto.pdf

Alcaldía de Bogotá. (1998a, 28 de agosto). Decreto 739 de 1998. https://www.alcaldiabogota.gov.co/sisjur/normas/ Norma1.jsp?i=1711

Alcaldía de Bogotá. (1998b, 10 de febrero). Decreto 176 de 1998. https://www.alcaldiabogota.gov.co/sisjur/normas /Norma1.jsp?i=2376

Alcaldía de Bogotá. (1999, 5 de agosto). Decreto 518 de 1999. https://www.alcaldiabogota.gov.co/sisjur/normas/No rma1.jsp?i=1755\&dt $=S$

Alcaldía de Bogotá. (2001, 30 de abril). Decreto 367 de 2001. https://www.alcaldiabogota.gov.co/sisjur/normas/No rma1.jsp?i $=3817 \& \mathrm{dt}=\mathrm{S}$

Alcaldía de Bogotá. (2002, 15 de marzo). Decreto 098 del 2002. https://legislacion.vlex.com.co/vid/decreto-098-44 9168050

Alcaldía de Bogotá. (2004, 23 de diciembre). Decreto 421 de 2004. https://www.alcaldiabogota.gov.co/sisjur/norma s/Norma1.jsp?i=15527\&dt=S

Alcaldía de Bogotá. (2005, 18 de abril). Decreto 124 de 2005. https://www.alcaldiabogota.gov.co/sisjur/normas/N orma1.jsp?i $=16301$

Alcaldía de Bogotá. (2006a, 31 de agosto). Decreto 342 de 2006. https://www.alcaldiabogota.gov.co/sisjur/normas/ Norma1.jsp?i=21504\&dt=S)

Alcaldía de Bogotá. (2006b). Acuerdo 257 de 2006. https://participacionbogota.gov.co/sites/default/files/2018-03/ Acuerdo-257-de-2006.pdf

Alcaldía de Bogotá. (2006c, 29 de diciembre). Decreto 539 de 2006. https://bogota.gov.co/sites/default/files/art/d c539.pdf

Alcaldía de Bogotá. (2006d). Acuerdo 265 de 2006.

Asamblea Nacional Constituyente. (1991a, 20 de julio). Gaceta Constitucional No. 116.

Asamblea Nacional Constituyente. (1991b). Constitución Política de Colombia 1991.

Botero, M. H., y Suárez E. C. (2010). Bogotá y la descentralización intraterritorial: crónica de una historia inconclusa. Universidad del Rosario.

Castro Castro, J. (1991). Bogotá, Distrito Capital. El Abedul. Gaceta Constitucional, 40, 37- 66.

Dangond, C., Jolly, J.-F., Monteoliva, A., y Rojas, F. (2011). Cuadernos de análisis y problemas urbanos en Colombia: la seguridad humana como eje transversal. Konrad Adenauer Stiftung.

Dangond, C., Jolly, J.-F., Monteoliva, A., y Rojas, F. (2013). Del transporte a la movilidad urbana en Bogotá: más que un problema de vías y automotores. Editorial Pontificia Universidad Javeriana.

Diario Oficial. (1992, 28 de enero). Ley 1 de 1992. Año CXXVII. No. 40307. 
Gobierno de Colombia. (1954, 17 de diciembre). Decreto 3640. http://www.suin-juriscol.gov.co/viewDocument.asp ?ruta $=$ Decretos $/ 1878195$

Gobierno de Colombia. (1986a, 1.. de enero). Acto Legislativo 01 de 1986. https://www.funcionpublica.gov.co/eva /gestornormativo/norma.php?i $=9440$

Gobierno de Colombia. (1986b, 25 de abril). Decreto 1333 de 1986. http://www.secretariasenado.gov.co/senado/b asedoc/decreto_1333_1986.html

Gobierno de Colombia. (1992, 1.. de enero). Ley 1 de enero 28 de 1992. http://www.suin-juriscol.gov.co/viewDocu ment.asp?id=1786120

Gobierno de Colombia. (1993, 21 de julio). Decreto 1421 De 1993. Estatuto Orgánico De Bogotá. http://www.subred sur.gov.co/content/decreto-1421-de-1993-estatuto-org\%C3\%A1nico-de-bogot\%C3\%A1

Gobierno de Colombia. (2000, 17 de agosto). Acto Legislativo 01 de 2000. http://www.suin-juriscol.gov.co/viewDo cument.asp?id $=30019387$

Gobierno de Colombia. (2004, 15 de julio). Ley 152 de 1994. http://www.secretariasenado.gov.co/senado/basedoc/ ley_0152_1994.html

Gobierno de Colombia. (2005, 2 de mayo). Decreto 1350 de 2005. https://www.funcionpublica.gov.co/eva/gestorn ormativo/norma.php?i=16332

Jolly, J.-F. (2010). Regir el territorio y gobernar los territorios. Políticas públicas de vivienda de interés social, servicios públicos domiciliarios y educación. Editorial Pontificia Universidad Javeriana.

Lemos Simmonds, C. (1991, 15 de marzo). Exposición de motivos del Distrito Especial de Bogotá. Gaceta Constitucional, 21, 64-88.

Montaño, D. C. (2016). El debate de la descentralización por localidades en Bogotá y una aproximación a la experiencia limeña. Lecciones y desafios para el Distrito Capital [trabajo de Grado para aspirar al título de Magíster en Análisis Problemas Políticos, Económicos e Internacionales Contemporáneos]. Instituto de Altos Estudios para el Desarrollo-Academia Diplomática San Carlos.

Muller, P. (2010). Las políticas públicas (3. a edición, J.-F. Jolly y V. C. Salazar, trads.). Universidad Externado de Colombia.

Nickson, A. (1998, 17-19 de abril). Where is Local Government going in Latin America? A comparative perspective. Conferencia Anual de la Sociedad de Estudios Latinoamericanos. Universidad de Liverpool.

Nieto, M. A. (2008). Descentralización subdistrital a la luz del estatuto orgánico de Bogotá: una mirada desde los ciclos de losproyectos locales [avance de trabajo de grado para optar para optar al título de Politóloga]. Pontificia Universidad Javeriana, Facultad de Ciencias Políticas y Relaciones Internacionales, Carrera de Ciencia Política, Bogotá.

Velásquez Gavilanes, R. (2003). Bogotá: politicas públicas de gobierno local. CEJA Centro Editorial Javeriano. Bogotá, Pontificia Universidad Javeriana.

\section{Notas}

* Artículo de investigación.

1 La redacción de este artículo ha tenido la colaboración de Diana Carolina Montaño Lopera, quien realizó, en el marco de la investigación Coherencia del estatuto orgánico de Bogotá y de los instrumentos de planeación y de gestión posterior a su expedición. Ilustración a partir de unos proyectos territoriales sectoriales seleccionados. Su Trabajo de Grado para aspirar al título de Magíster en Análisis Problemas Políticos, Económicos e Internacionales Contemporáneos del Instituto de Altos Estudios para el Desarrollo-Academia Diplomática San Carlos, fue titulado Descentralización a nivel local: un análisis al interior de Lima y Bogotá. Experiencias, lecciones y desafíos para el Distrito Capital, bajo la dirección de Jean-François Jolly (Montaño, 2016).

2 Coherencia del estatuto orgánico de Bogotá y de los instrumentos de planeación y de gestión posterior a su expedición. Ilustración a partir de unos proyectos territoriales sectoriales seleccionados (de aquí en adelante se citará como Coherencia...). Se trata de la tercera investigación que desarrolló el Grupo PU después de una sobre La seguridad humana en cuatro ciudades (Bogotá, Cali, Cartagena y Villavicencio. Dangond et al., 2011), y otra sobre La movilidad en Bogotá desde un enfoque de desarrollo humano (Dangond et al., 2013). 
3 El Artículo primero indica: "Colombia es un Estado social de derecho organizado en forma de República unitaria, descentralizada, con autonomía de sus entidades territoriales..." (cursivas agregadas).

4 Cabe indicar que mediante el Acto Legislativo 01 de 2000 (Gobierno de Colombia, 2000), se modificó el nombre de la capital. Desde ese momento se denominó "Bogotá, Capital de la República”.

5 De ahora en adelante se anotará como EOB.

6 Fue solamente a partir de la Ley 152 de 1994 (Gobierno de Colombia, 2004), relativo al Plan Nacional de Desarrollo, que se volvió obligatoria la elaboración y la posterior aprobación de un Plan de Desarrollo Distrital.

$7 \quad$ Las tres primeras razones las establecen los partidarios del modelo económico, y las tres siguientes los del modelo político.

8 Según Pierre Muller, "elaborar una política pública se reduce a construir una representación, una imagen de la realidad sobre la cual se quiere intervenir. Es en referencia a esta imagen cognitiva que los actores van a organizar su percepción del problema, confrontar sus soluciones y definir sus propuestas de acción: llamaremos este conjunto de imágenes 'el referencial de la política pública”' (Muller, 2010, p. 115).

9 Cabe señalar que Diana Carolina Montaño perteneció hasta octubre de 2006 a la Dirección de Seguimiento y Análisis Estratégico de la Secretaría Distrital de Gobierno, dependencia encargada de coordinar las relaciones con el Concejo de Bogotá y la Administración Distrital, en el que se hacía el seguimiento respectivo del trámite a los proyectos de acuerdo y sus discusiones en las sesiones del Concejo. La información que obtuvo es, entonces, de primera mano, al haber tenido la oportunidad de estar presente en todas las sesiones que abarcaban la discusión del proyecto de Acuerdo 135 y escuchar la intervención de concejales y de secretarios del despacho.

10 Según el Acuerdo 265 de 2006 (Alcaldía de Bogotá, 2006d), se trata de Gobierno, Seguridad, Hacienda, Planeación, Integración Social, Salud, Educación; Ambiente, Hábitat, Gestión Pública, Mujer, Movilidad, Desarrollo Económico y Cultura, Recreación y Deporte.

\section{Licencia Creative Commons CC BY 4.0}

Cómo citar este artículo: Dangond Gibsone, C., y Jolly, J.-F. (2020). A propósito de los 25 años del Estatuto Orgánico de Bogotá (EOB). Tres miradas sobre el modelo de descentralización de Bogotá como un modelo híbrido. Papel Político, 25. https://doi.org/10.11144/Javeriana.papo25.paeo 